\title{
CHARACTERISTICS OF TRANSVERSE WAVES IN CHROMOSPHERIC MOTTLES
}

\author{
D. Kuridze ${ }^{1,2}$, G. Verth ${ }^{3}$, M. Mathioudakis ${ }^{1}$, R. Erdélyi ${ }^{3}$, D. B. Jess ${ }^{1}$, R. J. Morton ${ }^{4}$, D. J. Christian ${ }^{5}$, and F. P. Keenan ${ }^{1}$ \\ ${ }^{1}$ Astrophysics Research Center, School of Mathematics and Physics, Queen's University, Belfast BT7 1NN, UK; dkuridze01@qub.ac.uk \\ ${ }^{2}$ Abastumani Astrophysical Observatory, Ilia State University, G. Tsereteli 3, 0612, Tbilisi, Georgia \\ ${ }^{3}$ Solar Physics and Space Plasma Research Center $\left(S^{2} \mathrm{RC}\right)$, University of Sheffield, Hicks Building, Hounsfield Road, Sheffield S3 7RH, UK \\ ${ }^{4}$ Mathematics and Information Science, Northumbria University, Camden Street, Newcastle Upon Tyne NE1 8ST, UK \\ ${ }^{5}$ Department of Physics and Astronomy, California State University, Northridge, CA 91330, USA \\ Received 2013 September 3; accepted 2013 October 11; published 2013 November 26
}

\begin{abstract}
Using data obtained by the high temporal and spatial resolution Rapid Oscillations in the Solar Atmosphere instrument on the Dunn Solar Telescope, we investigate at an unprecedented level of detail transverse oscillations in chromospheric fine structures near the solar disk center. The oscillations are interpreted in terms of propagating and standing magnetohydrodynamic kink waves. Wave characteristics including the maximum transverse velocity amplitude and the phase speed are measured as a function of distance along the structure's length. Solar magnetoseismology is applied to these measured parameters to obtain diagnostic information on key plasma parameters (e.g., magnetic field, density, temperature, flow speed) of these localized waveguides. The magnetic field strength of the mottle along the $\sim 2 \mathrm{Mm}$ length is found to decrease by a factor of 12 , while the local plasma density scale height is $\sim 280 \pm 80 \mathrm{~km}$.
\end{abstract}

Key words: magnetohydrodynamics (MHD) - Sun: atmosphere - Sun: chromosphere - Sun: magnetic fields Sun: oscillations

Online-only material: color figures

\section{INTRODUCTION}

Chromospheric fine-scale structures such as limb spicules, on-disk mottles, and dynamic fibrils are among the most popular objects for study in solar physics today. These jet-like plasma features, formed near the network boundaries, can protrude into the transition region and low corona (Beckers 1968, 1972; Sterling 2000; De Pontieu \& Erdélyi 2006) and act as conduits for channeling energy and mass from the solar photosphere into the upper solar atmosphere and the solar wind (De Pontieu et al. 2004; De Pontieu \& Erdélyi 2006; Morton 2012).

Recent ground-based and space-borne observations have shown a plethora of waves and oscillations in these structures (Kukhianidze et al. 2006; Zaqarashvili et al 2007; De Pontieu et al. 2007; He et al. 2009a, 2009b; Zaqarashvili \& Erdélyi 2009; Okamoto \& de Pontieu 2011; Kuridze et al. 2012; Morton et al. 2012; Mathioudakis et al. 2013). These oscillations are usually observed as periodic transverse displacements (Zaqarashvili \& Erdélyi 2009; Okamoto \& de Pontieu 2011; Pietarila et al. 2011; Kuridze et al. 2012; Morton et al. 2012). The observations support the idea that the chromospheric fine structures can be modeled as thin, overdense magnetic flux tubes that are waveguides for the transverse oscillations with periods that have an observational upper bound limited by their finite visible lifetime. This is also supported by three-dimensional numerical modeling of the chromosphere (Leenaarts et al. 2012). In this regard, the observed transverse oscillations have been interpreted as fast kink MHD waves (Spruit 1982; Erdélyi \& Fedun 2007).

Despite a number of recent advances in the field, a detailed study of the properties and physical nature of the chromospheric fine structures remains a challenging observational task. The propagating or standing nature of the oscillations are crucial to understanding the role of these waves in the energy balance of the solar atmosphere and their contribution to the atmospheric heating process. Both propagating (upward and downward) and standing transverse oscillations have been reported in limb spicules (Zaqarashvili et al 2007; Zaqarashvili \& Erdélyi 2009; He et al. 2009a, 2009b; Okamoto \& de Pontieu 2011). In addition, a few instances of propagating oscillations were also recorded in chromospheric mottles (Kuridze et al. 2012; Morton et al. 2012), which are believed to be the disk counterparts of limb spicules (Tsiropoula \& Schmieder 1997; Zachariadis et al. 2001; Hansteen et al. 2006; Scullion et al. 2009; Rouppe van der Voort et al. 2009).

The study of MHD wave properties in spicular structures opens new dimensions to chromospheric plasma diagnostics using the tools of solar magnetoseismology (SMS), a field that has recently emerged (for reviews on SMS see, e.g., Erdélyi 2006; Andries et al. 2009; Ruderman \& Erdélyi 2009; Taroyan \& Erdélyi 2009). One of the techniques developed estimates the variation of magnetic field strength and plasma density along the chromospheric magnetic flux tube from the properties of kink waves (Verth et al. 2011). This approach has been successfully applied to Hinode/SOT Ca II H limb spicule observations (Verth et al. 2011).

In this paper, we present results on transverse oscillations observed in the $\mathrm{H} \alpha$ on-disk and quiet-solar chromospheric mottles. Our work provides evidence for upward and downward propagating and standing waves. In the case of the propagating sample, wave characteristics such as maximum transverse velocity amplitude and phase speed are measured as a function of distance along the structures length. The wave properties are used to estimate plasma parameters along the waveguide by employing the SMS approach.

\section{OBSERVATIONS AND DATA REDUCTION}

Observations were undertaken between 13:46 and 14:40 UT on 2009 May 28 at disk center with the Rapid Oscillations in the Solar Atmosphere (ROSA; Jess et al. 2010a) imaging system, and with the Interferometric Bidimensional Spectrometer (IBIS; Cavalini 2006), mounted at the Dunn Solar Telescope (DST) at the National Solar Observatory, NM, USA. The 


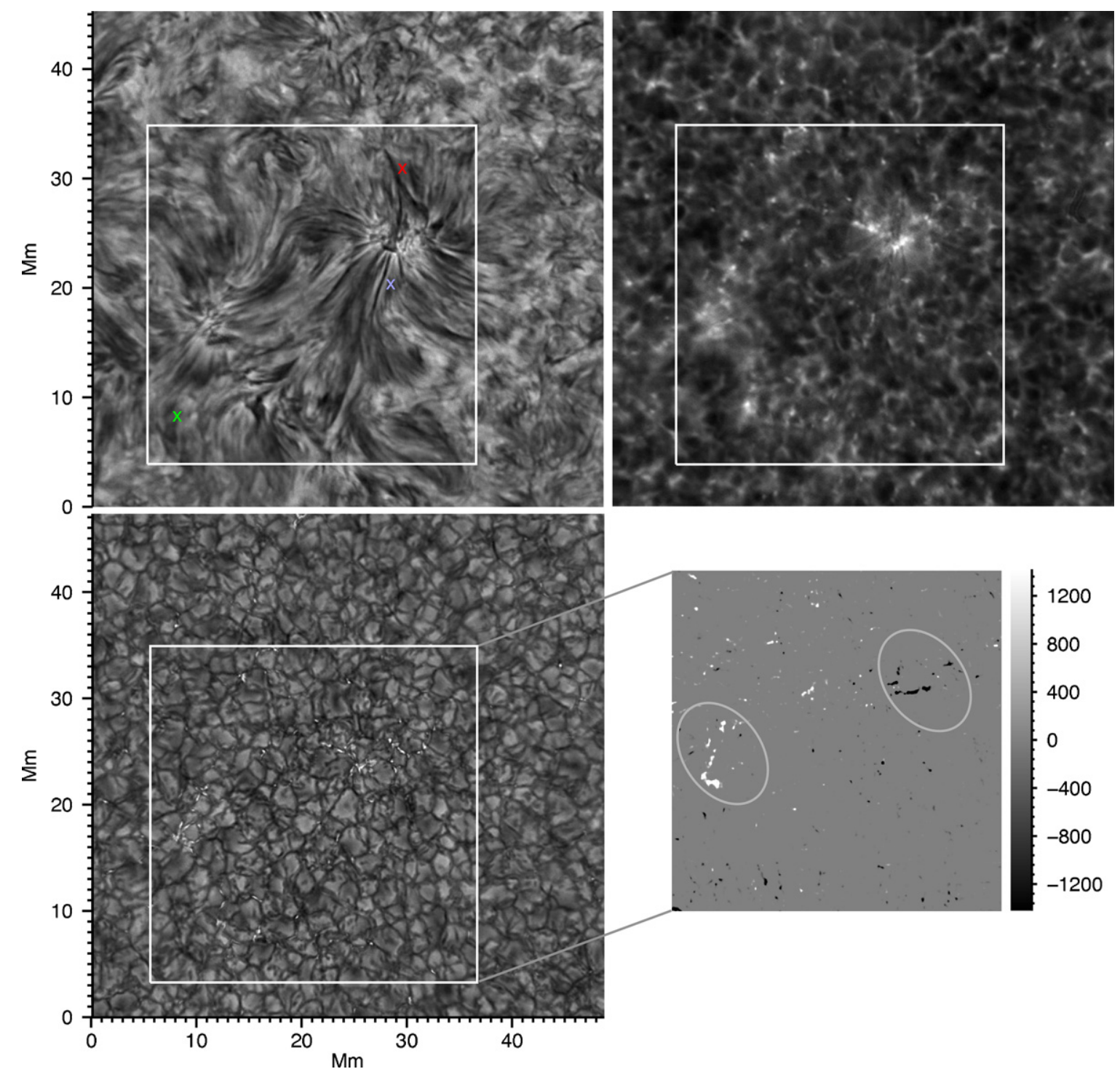

Figure 1. Co-spatial and co-temporal ROSA images in the $\mathrm{H} \alpha$ core (top left), the Ca II K core (top right), and the $G$ band (bottom left) together with an LOS magnetogram (bottom right). The rosettes where the mottles are concentrated are highlighted with white boxes. The crosses in the H $\alpha$ image indicate the positions of the mottles selected for the analysis. The ellipses in the LOS magnetogram denote patches of magnetic flux concentrations, with the color scale indicating the magnetic field strength in gauss. The $\mathrm{H} \alpha$ image shows that the footpoints of the chromospheric fine structures correspond to Ca II $K$ - and $G$-band bright points and strong magnetic flux concentrations that highlight the boundaries of the supergranular cell.

(A color version of this figure is available in the online journal.)

ROSA dataset includes simultaneous imaging in the $\mathrm{H} \alpha$ core at $6562.8 \AA$ (bandpass $0.25 \AA$ ), the Ca II K core at $3933.7 \AA$ (bandpass $1.0 \AA$ ), the $G$ band at $4305.5 \AA$, bandpass $(9.20 \AA$ ), and the line-of-sight (LOS) magnetograms. High-order adaptive optics were applied throughout the observations (Rimmele 2004). The images were reconstructed by implementing the speckle algorithms of Wöger et al. (2008) followed by de-stretching. These algorithms have removed the effects of atmospheric distortion from the data. The effective cadence after reconstruction is reduced to $4.2243 \mathrm{~s}$ for $\mathrm{H} \alpha$ and $\mathrm{Ca}$ II $\mathrm{K}$. Observations were obtained with a spatial sampling of 0.069 pixel $^{-1}$ corresponding to a spatial resolution of $0^{\prime \prime} .21$ over the $62^{\prime \prime} \times 62^{\prime \prime}$ field of view (FOV).

LOS magnetograms were constructed using the left- and right-hand circularly polarized light obtained $125 \mathrm{~m} \AA$ into the blue wing of the magnetically sensitive Fe I line at $6302.5 \AA$. A blue-wing offset was required to minimize granulation contrast, while conversion of the filtergram into units of gauss was performed using simultaneous $\mathrm{SOHO} / \mathrm{MDI}$ magnetograms (see discussion in Jess et al. 2010b).

IBIS undertook simultaneous $\mathrm{NaI} \mathrm{D}_{1}$ core imaging at $5895.94 \AA$ with a spatial sampling of $00^{\prime} 083$ pixel $^{-1}$ over the same FOV. The IBIS data have a post-reconstruction cadence of 39.7 s. Despite difficulties in interpreting the $\mathrm{Na}_{\mathrm{I}} \mathrm{D}_{1}$ line formation height, it is suggested that it is formed in the upper photosphere/lower chromosphere (Eibe et al. 2001; Finsterle et al. 2004). Doppler wavelength shifts of the $\mathrm{Na}$ I $\mathrm{D}_{1}$ line profile minimum were used to construct LOS velocity maps of the same FOV (for more details see Jess et al. 2010b).

\section{RESULTS AND ANALYSIS}

Figure 1 shows co-spatial and co-temporal snapshots in the $\mathrm{H} \alpha$ core and in $\mathrm{Ca}$ II $\mathrm{K}$, and the $G$ band, where the FOV covers a quiet Sun region near disk center. The $\mathrm{H} \alpha$ image contains a large rosette structure located near the center (see the top 

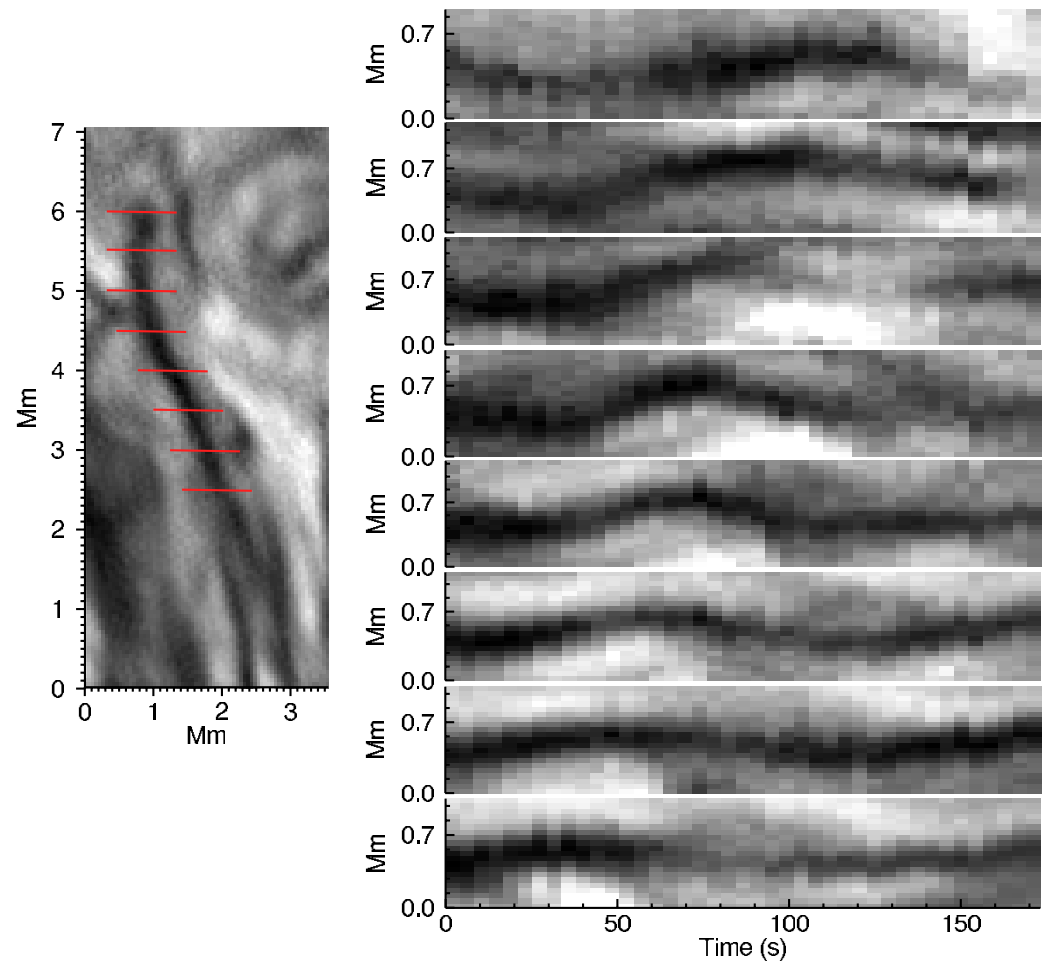

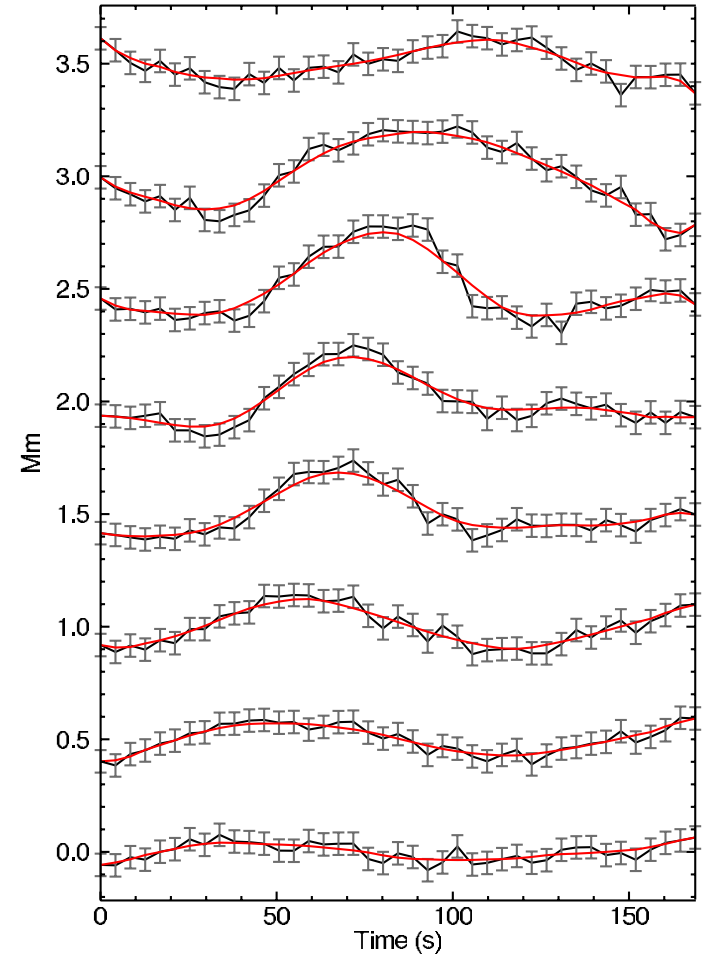



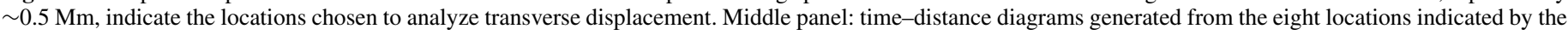

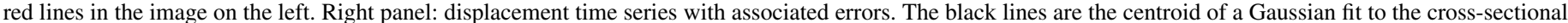
flux profiles of the mottle at each time frame of the transverse cross cuts smoothed by a $\sim 25 \mathrm{~km}$ width (red lines).

(A color version of this figure is available in the online journal.)

left panel of Figure 1). Rosettes are clusters of elongated, dark $\mathrm{H} \alpha$ mottles expanding radially around a common center over internetwork regions (Zachariadis et al. 2001; Tziotziou et al. 2003; Rouppe van der Voort et al. 2007). Three additional smaller rosettes are visible in the lower left of the boxed area (the top left panel of Figure 1). The roots of the rosettes are co-spatial with Ca II K brightenings, $G$-band bright points, and strong magnetic field concentrations that outline the boundaries of the supergranular cell highlighted with the dashed box in Figure 1. An LOS magnetogram of the FOV shows that the supergranular cell boundary consist of opposite polarity magnetic field concentrations (bottom right panel of Figure 1).

The application of time-distance $(t-d)$ analysis to the $\mathrm{H} \alpha$ images reveals that the mottles display transverse motions perpendicular to their axis, usually interpreted as transverse MHD kink motions (Spruit 1982; Edwin \& Roberts 1983; Erdélyi \& Fedun 2007). Periodic transverse displacements of three different mottles, marked with crosses in Figure 1, have been selected for further analysis.

Figure 2 shows a more detailed view of the mottle located near the red cross in Figure 1. The projected length of the structure is $\sim 4 \mathrm{Mm}$, with a resolved average width of $350 \mathrm{~km}$ and lifetime of $\sim 3$ minutes. The red lines across the structure indicate the locations of the cross cuts used to study the transverse oscillations. We use the cross cuts to generate two-dimensional $t-d)$ diagrams that reveal the transverse motions at each point along the mottle's length. These $t-d$ samples are plotted in the middle panel of Figure 2. The shift detected in the signal, as a function of time, indicates that the observed oscillation is due to a propagating wave. Displacements are determined by fitting a Gaussian function to the cross-sectional flux profile for each time frame of the transverse cross cuts (right panel of Figure 2). This method can determine the position of the structure's centroid to within 1 pixel and thus has an error of $\pm 50 \mathrm{~km}$. It should be noted that in Figure 2 there are only eight cross cuts (separated by $0.5 \mathrm{Mm}$ ), the corresponding $t-d$ diagrams and time series, respectively. We note that we generated and analyzed the $t-d$ diagrams for 15 cross cuts separated by $0.25 \mathrm{Mm}$ but we chose to show here only eight of those for presentation purposes. A linear trend was subtracted from the displacement time series to obtain the periodic motions. The time series was fitted with a harmonic function at each position along the mottle from which the periods of the wave are derived with a median value of $P \approx 120 \pm 10 \mathrm{~s}$.

We measured the maximum transverse displacements at each of the 15 positions along the $\sim 3.7 \mathrm{Mm}$ mottle length (left panel of Figure 2). The maximum transverse velocity amplitudes are derived using $v_{\perp}=2 \pi \xi_{\perp} / P$, where $\xi_{\perp}$ and $P$ are the maximum transverse displacement and period of the oscillation, respectively. Uncertainties in $v_{\perp}$ are estimated from the error in $\xi_{\perp}$ and the standard deviation of $P$. The results with the associated error of each data point are plotted in the left panel of Figure 3 . This figure clearly shows that the velocity amplitude is increasing up to around $2 \mathrm{Mm}$ (Figure 3) and then decreases. Due to the different trends, the first eight and last seven data points are fitted separately by an exponential function of the form $v_{\perp}(s)=v_{\perp, 0} \exp \left(s / A_{2}\right)$, where $s$ is the distance along the mottle. We note that the exponential function has the best fit (with $95 \%$ confidence level) for the first eight data points. We obtain $v_{\perp, 0}=2.5 \pm 1.0 \mathrm{~km} \mathrm{~s}^{-1}$ and $A_{2}=1.12 \pm 0.35 \mathrm{Mm}$ for $s \leqslant 1.75 \mathrm{Mm}$ and $A_{2}=-5.3 \pm 4 \mathrm{Mm}$ for $s>1.75 \mathrm{Mm}$. Errors in these fitting parameters are their $1 \sigma$ uncertainties derived 

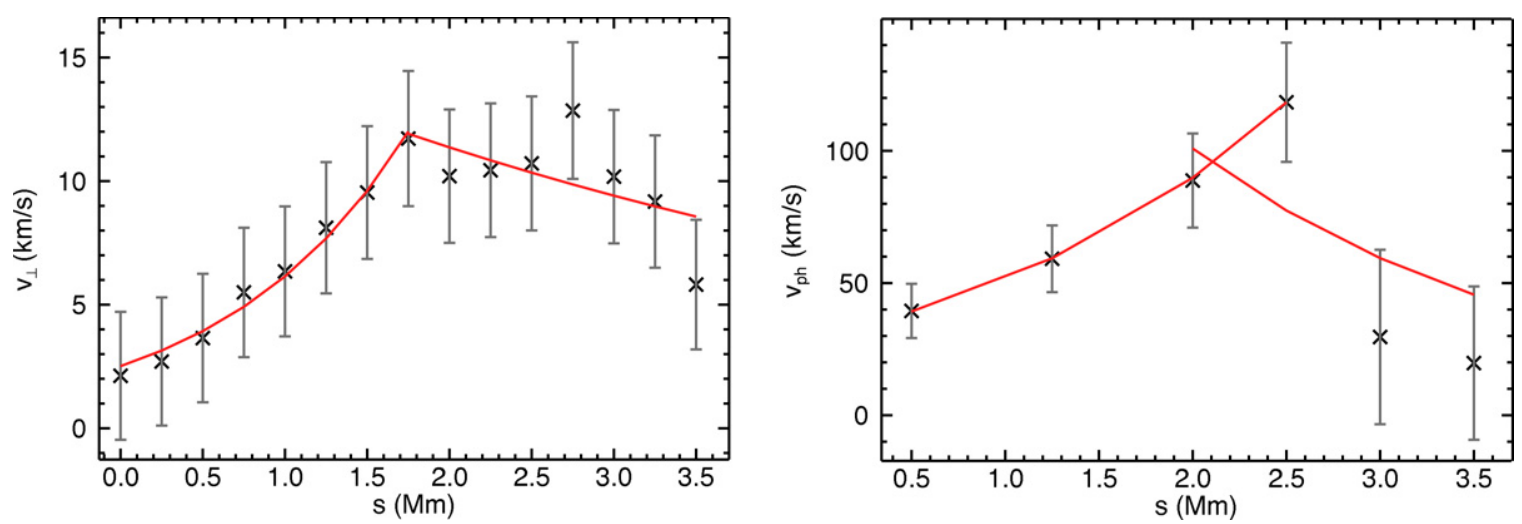

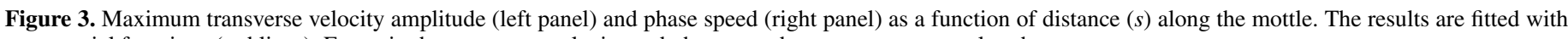
exponential functions (red lines). Errors in the transverse velocity and phase speed measurements are also shown.

(A color version of this figure is available in the online journal.)

from the fitting algorithm, which use the measurement error for each data point.

The phase speed of the transverse motions can be evaluated from the time delay in the signals obtained at different positions along the mottle. From the displacement time series, we can measure the time coordinates of the maximum transverse displacements. A phase speed between two positions along the structure can be calculated as $v_{\mathrm{ph}}=L / t_{l}$, where $L$ is the distance between two selected heights and $t_{l}$ is the time delay between the location of the maximum displacements. The time at the location of the maximum transverse displacement in the time series can be estimated to within one temporal resolution element of $4.224 \mathrm{~s}$, and the maximum phase speed, which can be resolved for the length $L$ along the structure, is $v_{\mathrm{ph}_{\max }} \sim L / 4.224$. We determine a reliable phase speed of the transverse wave for six consecutive segments along the mottle length, and the results (with corresponding measurement errors) are plotted in the right panel of Figure 3. The phase speed is $\sim 40 \mathrm{~km} \mathrm{~s}^{-1}$ near the lower part of the mottle and increases to $\sim 120 \mathrm{~km} \mathrm{~s}^{-1}$ at $2.5 \mathrm{Mm}$, and then it decreases again toward the end of the structure (right panel of Figure 3). We fit the data points using an exponential function of the form $v_{\mathrm{ph}}(s)=v_{\mathrm{ph}, 0} \exp \left(-s / A_{1}\right)$ with $v_{\mathrm{ph}, 0}=29.7 \pm 6.7 \mathrm{~km} \mathrm{~s}^{-1}, A_{1}=-1.81 \pm 0.24 \mathrm{Mm}$ for $s \leqslant 2.5 \mathrm{Mm}$ and $A_{2}=1.88 \pm 0.3 \mathrm{Mm}$ for $s>2.5 \mathrm{Mm}$.

Figure 4 shows the $\mathrm{H} \alpha$ mottle (left panel) located near the green cross of Figure 1 and its transverse displacements at different positions along its length (middle and right panels of Figure 4). Time series, obtained using a method similar to that described for the first mottle in Figure 2, highlight the upward and downward propagating waves with a period of $P \approx 180 \pm 10 \mathrm{~s}$ (see the green and blue diagonal lines in the right panel of Figure 4). The average phase speed and maximum transverse velocity along the length of the mottle are $101 \pm 14 \mathrm{~km} \mathrm{~s}^{-1}, 8.8 \pm 3.1 \mathrm{~km} \mathrm{~s}^{-1}, 79 \pm 8 \mathrm{~km} \mathrm{~s}^{-1}$, and $11.4 \pm 3.3 \mathrm{~km} \mathrm{~s}^{-1}$ for the upward and downward propagating waves, respectively. Unfortunately, for this example, large uncertainties in the transverse velocity and phase speed measurements do not allow us to study their variation as a function of distance.

Figure 5 shows the chromospheric structure marked with a blue cross in Figure 1 and its transverse displacement with a period of $P \approx 131 \pm 15 \mathrm{~s}$. We detect a marginal delay of about $10 \mathrm{~s}$ in the oscillation signals at the lower and upper positions (Figure 5). This time delay, combined with a distance of around 3.5 $\mathrm{Mm}$ between these positions (see left panel of Figure 4), gives a propagating speed of more than $350 \mathrm{~km} \mathrm{~s}^{-1}$, which is too high for what might be expected for the phase speed of the kink waves in chromospheric mottles. We believe that this high speed may be caused by the standing wave pattern generated by the superposition of two oppositely directed waves.

\section{MAGNETOSEISMOLOGICAL DIAGNOSTICS}

A novel SMS tool that allows us to determine the variation of the magnetic field and plasma density along a chromospheric structure using the characteristics of kink oscillations has been developed by Verth et al. (2011). Based on their approach, if the kink speed $v_{\mathrm{ph}}(s)$ (which is the phase speed of the kink wave) and maximum transverse velocity amplitude $v_{\perp}(s)$ are estimated from observations, then the expansion rate of the magnetic flux tubes can be derived from the solution of the kink wave governing equation (see Equation (1) of Verth at al. 2011). The flux tube radius as a function of $s$ is given by

$$
R(s)=R(0) \exp \left(\frac{s}{H}\right)
$$

where $H=2 A_{1} A_{2} /\left(2 A_{1}+A_{2}\right)$ and $A_{1}, A_{2}$ are fitting parameters defined from the measured $v_{p h}(s)$ and $v_{\perp}(s)$, and $R(0)$ is the flux tube radius at the lowest position (Verth et al. 2011). On the other hand, from magnetic flux conservation $B(s) \propto 1 / R^{2}(s)$, where $B(s)$ is the average magnetic field strength and the variation of the magnetic field along the flux tube can be estimated. Furthermore, from the kink speed and magnetic field variations, the plasma density along the structure can also be determined using

$$
\rho(s) \propto \frac{B^{2}(s)}{v_{\mathrm{ph}}^{2}(s)},
$$

where $\rho(s)$ is the average of the internal and external plasma densities (see Verth et al. 2011 for further details).

Inspired by this work, we estimate these parameters for the on-disk mottle presented in Figure 2 using the same SMS tool. We employ the functions of $v_{\mathrm{ph}}(s)$ and $v_{\perp}(s)$ found in the previous section using the exponential fit for the measured phase travel time and velocity amplitudes. From these functions, which define $A_{1}$ and $A_{2}$, and from Equation (1), we make a piecewise estimate of the normalized area expansion of the flux tube. The magnetic field variation can also be evaluated from the area expansion and magnetic flux conservation law (middle panel of Figure 6). Furthermore, the normalized plasma density along 

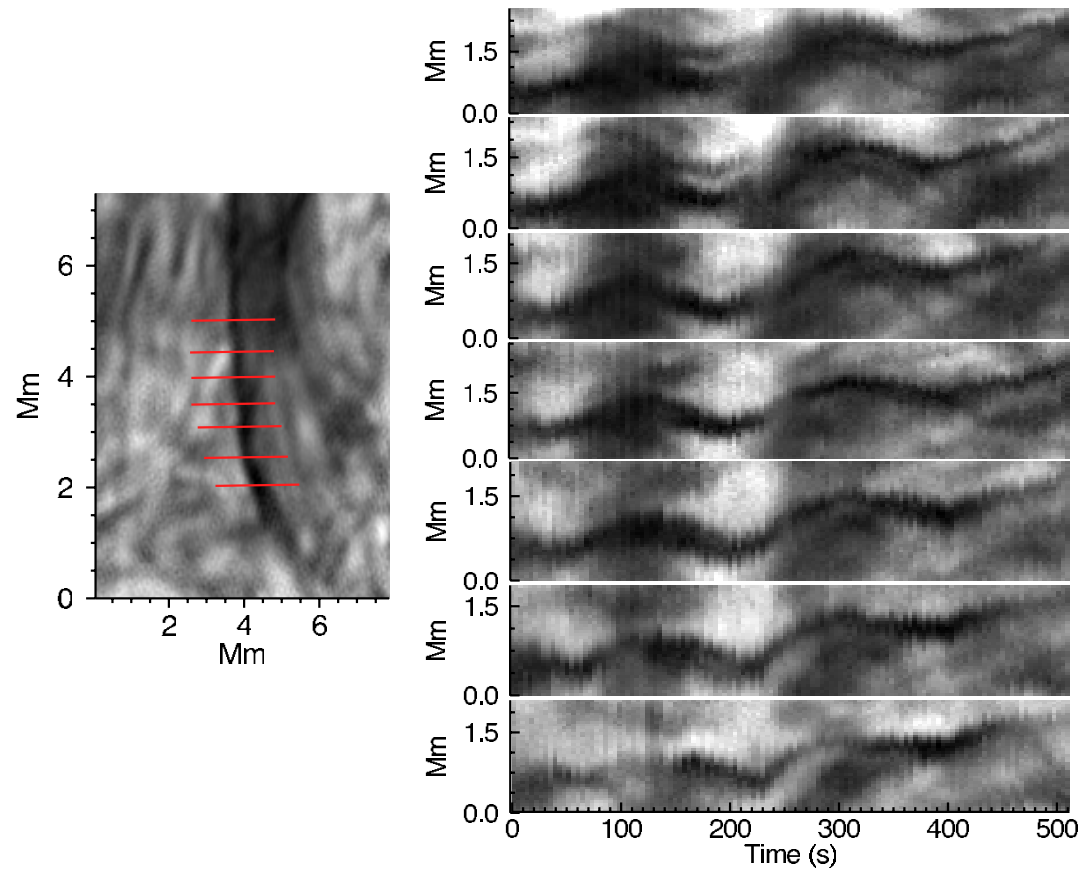

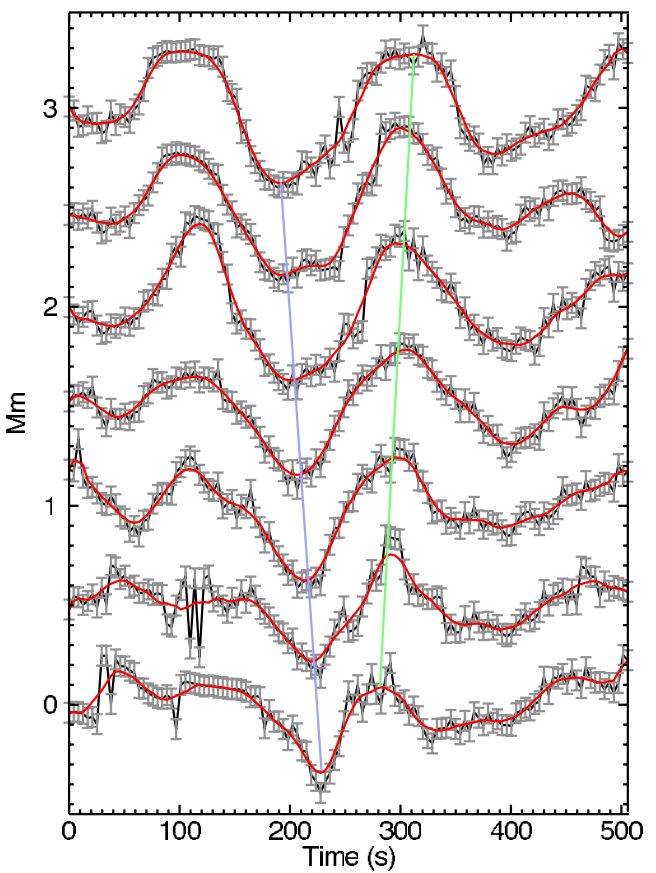

Figure 4. Left panel: detailed view of the mottle from its bottom to top in the image plane located near the green cross in Figure 1 . The red short lines separated by $\sim 0.5 \mathrm{Mm}$ indicate the locations on the mottles where time-distance plots depicted on the middle panel (from bottom to top) are generated. Right panel: displacement time series with error bars. The diagonal lines highlight the downward (blue) and upward (green) propagating motions.

(A color version of this figure is available in the online journal.)
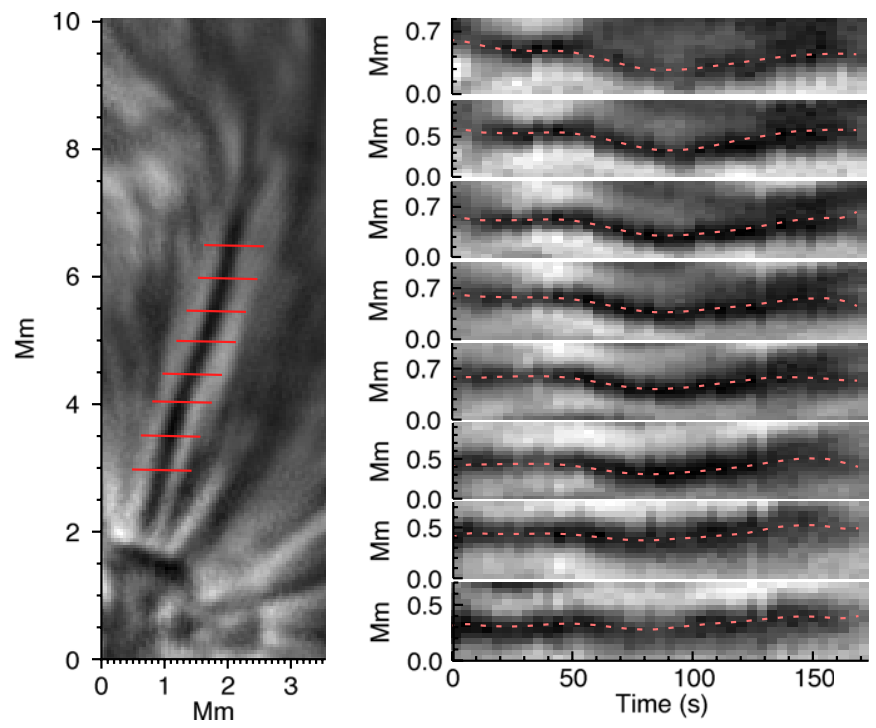

Figure 5. Mottle from its bottom to top in the image plane located near the blue cross in Figure 1. Cross cuts, separated by $\sim 0.5 \mathrm{Mm}$, indicate the locations on the mottle where the displacement is further analyzed (left panel). The pink dotted lines are the centroids of a Gaussian fit to the cross-sectional flux profiles of the mottle at each time step (right panel).

(A color version of this figure is available in the online journal.)

this mottle, estimated from Equation (2), is shown in the right panel of Figure 6.

\section{DISCUSSION AND CONCLUSIONS}

Several observational and theoretical studies suggest that the transverse MHD kink waves observed in chromospheric structures can be excited by granular buffeting, global oscillations, mode conversion, or torsional motions in the photospheric bright points where chromospheric fine structures are anchored
(Roberts 1979; Spruit 1981; Hollweg 1981; Hasan \& Kalkofen 1999; De Pontieu et al. 2004; Jess et al. 2012; Morton et al. 2013). By tracking the oscillation signals at different positions, we detected both propagating and standing wave modes along chromospheric mottles that appear to be rooted in regions with strong magnetic field concentrations (Figure 1). Upward propagating waves with periods of $\sim 120 \mathrm{~s}$ are detected in one of the mottles (Figure 2). The analysis shows that the phase speed and transverse velocity amplitude rise exponentially with distance along the mottle length up to about $2.5 \mathrm{Mm}$ before they begin to decrease (Figure 3). MHD wave theory suggests that the variation of these wave characteristics is controlled by changes in plasma parameters such as density and magnetic field strength along the waveguide. A decrease in the plasma- $\beta$ will result in the mottle plasma parameters gradually becoming dominated by the magnetic field, causing the observed growth of the phase speed (Figure 3). At a height of around 2-2.5 Mm, the magnetic canopy is formed (Solanki \& Steiner 1990; Wedemeyer-Böhm et al. 2009; Tsiropoula et al. 2012). This is the layer where the gas and magnetic pressures are equal $(\beta \approx 1)$ and where the mode conversion through, e.g.,, nonlinear interactions, can occur (Rosenthal et al. 2002; Bogdan et al. 2003; Hasan et al. 2003; Schunker \& Cally 2006; Kuridze et al. 2008). The amplitude of the transverse motions increases, and at about $2 \mathrm{Mm}$ reaches $250 \mathrm{~km}$ (Figure 2), which is similar to the waveguide width of $350 \mathrm{~km}$. Hence, the observed fast kink wave mode may become nonlinear near the canopy area, which may lead to mode conversion, and thus energy transfer between nonlinear kink modes and longitudinal waves. This can result in the observed decrease of phase speed and transverse velocity at a higher length along the structure (Figure 3).

Waves that propagate from the lower chromosphere into the transition region may undergo reflection at the top of the canopy due to the sharp density gradient (Hollweg et al. 1982; Rosenthal et al. 2002; Kuridze et al. 2008; Fujimura \& Tsuneta 2009). The 

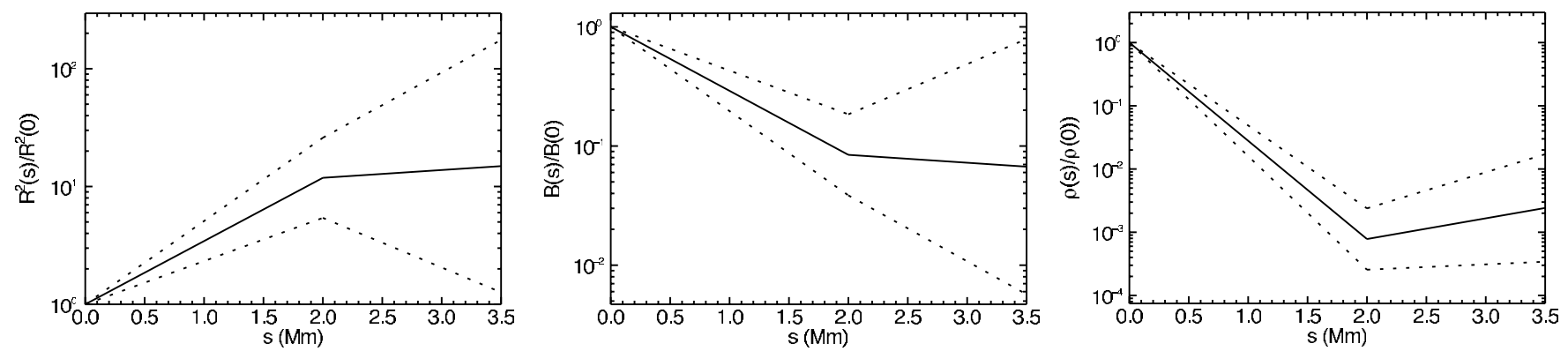


as a function of length along the waveguide shown in Figure 2 . The dotted lines indicate the region of uncertainty due to the $1 \sigma$ error of $A_{1}$ and $A_{2}$.

conditions for the reflection of the kink waves are defined by the local cut off period, the highest period that is allowed to propagate. Following the Kneser oscillation theorem (Kneser 1893), if the phase speed is increasing with height, then the governing kink wave equation processes a cut off that can be calculated as $P_{c} \approx 4 \pi \Delta s / \Delta v_{\mathrm{ph}}$, where $\Delta v_{\mathrm{ph}}$ is the change of the phase speed as a function of distance $s$ and $\Delta s$ is the distance between two selected points. For the waveguide presented in Figure 2 this corresponds to a cut off period of $\sim 314 \mathrm{~s}$. This value is much higher than the observed kink wave period ( $\sim 120 \mathrm{~s})$, suggesting that the observed wave, and waves with periods less then the estimated cut off, should propagate into the upper chromospheric layers without reflection. The high cut off period indicates that the chromospheric mottles could allow the propagation of long period ( $\mathrm{P}>3$ minutes) transversal (kink and Alfvén) waves as well. Those waves are observed in the corona and are thought to be an important contributor to the coronal heating, at least in the case of the quiet Sun (e.g., McIntosh et al. 2011).

It appears that the upward- and downward-directed waves (the green and blue diagonal lines in the right panel of Figure 4) have an approximately constant phase speed $\left(\Delta v_{\mathrm{ph}} \approx 0\right)$ along the $\sim 3 \mathrm{Mm}$ mottle length. Almost constant phase speeds for torsional waves were also detected along limb spicules (see, e.g., De Pontieu et al. 2012; Sekse et al. 2013). A constant phase speed suggests that there is no cut off period, i.e., waves of any period can propagate along those fine-scale structures in the chromosphere. However, the downward propagating wave detected along the mottle presented in Figure 4 may be formed as a result of reflection of the upward propagating wave at the transition region boundary or in the corona. Furthermore, for this mottle we measured $v_{\text {up }} \approx 101 \mathrm{~km} \mathrm{~s}^{-1}$ and $v_{\text {down }} \approx 79 \mathrm{~km} \mathrm{~s}^{-1}$, where $v_{\text {up }}$ and $v_{\text {down }}$ are the phase speeds of upward and downward propagating waves, respectively. This difference could be a result of plasma flow along the mottle. In the presence of flow, the upward and downward kink speeds are modified by the flow as follows,

$$
\begin{gathered}
v_{\text {up }}=v_{\mathrm{ph}}+U, \\
v_{\text {down }}=v_{\text {ph }}-U,
\end{gathered}
$$

where, $v_{\mathrm{ph}}$ is the kink speed for the mottle with no flow and $U$ is the flow speed. This suggests a plasma flow along this mottle of $U \approx 11 \mathrm{~km} \mathrm{~s}^{-1}$ in the upward direction. A snapshot of the LOS velocity map of the studied region, obtained from Doppler wavelength shifts of the $\mathrm{Na}$ I $\mathrm{D}_{1}$ profile, shows that the lower chromosphere is dominated by the flow patterns (Figure 7). Values of the flow speed vary from around $-4.2 \mathrm{~km} \mathrm{~s}^{-1}$ (upward) to $7 \mathrm{~km} \mathrm{~s}^{-1}$ (downward) with an error

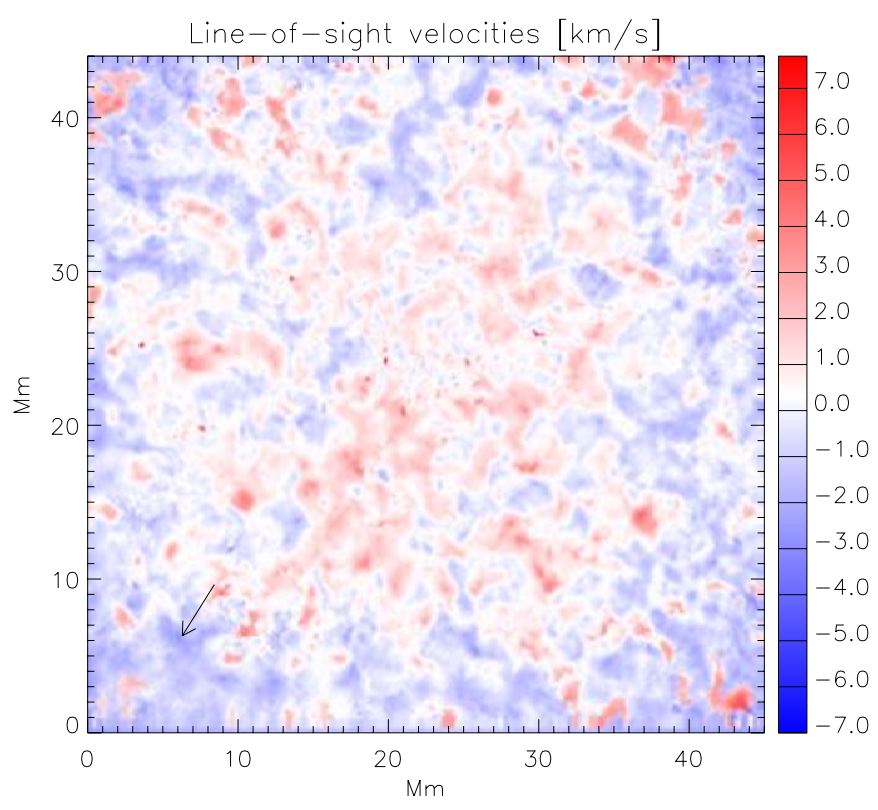

Figure 7. Snapshot of the LOS velocity map of the full FOV. The red and blue colors represent positive Doppler velocities (downflows) and negative Doppler velocities (upflows), respectively. The arrow indicates the position of the mottle analyzed in Figure 4.

(A color version of this figure is available in the online journal.)

of $\pm 0.5 \mathrm{~km} \mathrm{~s}^{-1}$ (Jess et al. 2010b). The average upflow LOS velocity near the footpoint of this mottle is around $-4 \mathrm{~km} \mathrm{~s}^{-1}$ (Figure 7). This value is consistent with the seismologically estimated phase speed, $\sim 10 \mathrm{~km} \mathrm{~s}^{-1}$, which is the horizontal component, and thus may be higher depending on the structure's inclination. Recently, Vissers \& Rouppe van der Voort (2012) measured upflow/downflow velocities within the range of -5.7 to $+13.5 \mathrm{~km} \mathrm{~s}^{-1}$ in chromospheric fibrils, consistent with our observations and SMS estimations.

A superposition of the opposite-directed kink waves may result in a wave with a very high phase speed that can be considered to be a (partially) standing wave (Fujimura \& Tsuneta 2009). The phase speed of the transverse wave shown in Figure 5 is $\sim 350 \mathrm{~km} \mathrm{~s}^{-1}$. This value is considerably higher than the local Alfvén/sound/kink speeds, indicating that it may be the consequence of the superposition of up and down propagating waves.

In Figure 6, we show the normalized estimated area expansion, magnetic field, and plasma density variations as functions of length along the waveguide shown in Figure 2. The area expansion factor along the $\sim 2 \mathrm{Mm}$ flux tube length is found to be $\sim 12$ (left panel of Figure 6), with a decrease in the magnetic 
field strength of the same factor (middle panel of Figure 6). Unfortunately, even modern high-resolution observations cannot yet provide direct, precise measurements of the flux tube expansion rate and magnetic field variation from the photosphere to chromosphere. Thus, it is very difficult to compare the results obtained from magnetoseismological techniques with direct measurements. We emphasize that spectropolarimetric measurement of chromospheric spicular magnetic field strengths of $B_{0} \sim 10-50$ G (Trujillo Bueno et al. 2005; Centeno et al. 2010), and the observed footpoint photospheric magnetic field strength of $\sim \mathrm{kG}$ (lower right panel of Figure 1) give comparable factors for the decrease in the field strength.

It must be noted that the $\mathrm{H} \alpha$ image of the mottle (left panel of Figure 6) does not show visual expansion by a factor of 12 (which would correspond to a radius change by a factor of 3.5). A Gaussian fit to the cross-sectional flux profile of the mottle suggests that the width of this structure at around $2 \mathrm{Mm}$ at its length (left panel of Figure 6) is $\sim 350 \mathrm{~km}$. Hence, according to our SMS estimation, the width at its base is expected to be $\sim 100 \mathrm{~km}$. This is the typical diameter of the $G$-band bright points that are considered as the footpoints of the mottles (Crockett et al. 2010). However, $100 \mathrm{~km}$ is below the spatial resolution of the ROSA H $\alpha$ filter. DeForest (2007) has suggested that the solar threadlike structures' expansion may not be seen visually (through imaging observations) if the threads have a sub-resolution width. The chromospheric fine structures analyzed in this paper are near the resolution limit, which could be the reason for the relatively constant apparent width along their length.

The normalized plasma density from our magnetoseismological study (plotted in the right panel of Figure 6) shows that the density along the mottle decreases by $10^{3}$ in a $2 \mathrm{Mm}$ length. Despite such a significant drop in density, the mottle is still visible in the $\mathrm{H} \alpha$ images. For a resolved dense flux tube in the chromosphere, the intensity is proportional to density, opacity, geometric depth, and the source function. The source function gives the contribution that the plasma makes to the intensity due to absorption/emission and cannot be determined directly from observation. With respect to unresolved (or near resolution) flux tubes, there are added complications. DeForest (2007) investigated the effect of geometric expansion on intensity for such structures. He found that the effect of sub-resolution flux tube expansion results in an apparent constant flux tube width and enhanced brightness with height (see Figure 4 of DeForest 2007). This simple geometric effect could be true for the dark, absorption $\mathrm{H} \alpha$ mottles as well, and hence it may explain why the upper part of the mottle is visible in $\mathrm{H} \alpha$. In addition, we would like to emphasize that the density of $\mathrm{H} \alpha$ dark mottles estimated by previous works (see, e.g., Tsiropoula \& Schmieder 1997 ) is about $10^{10} \mathrm{~cm}^{-3}$. For this value, our SMS density diagnostic method suggests that near the base the density would be approximately $10^{13} \mathrm{~cm}^{-3}$, which is a realistic value according to different atmospheric models (see, e.g., Vernazza et al. 1981; Fontenla et al. 2007).

Density diagnostics provide an estimate for the local plasma density scale height of $H_{\rho}=(280 \pm 80) \mathrm{km}$ along the $2 \mathrm{Mm}$ length, which is lower than some previous seismological estimates of the scale height $(\sim 700 \mathrm{~km})$ in limb spicules (Verth et al. 2011; Makita 2003). The density scale height could be used to estimate the mottle temperature in the isothermal approximation using $H_{\rho} \approx[T /(1 \mathrm{MK})] 47 \mathrm{Mm}$ (see, e.g., Aschwanden 2004). This yields $T=(5957 \pm 1702) \mathrm{K}$ for the mottles presented in Figure 4. The earlier work of Giovanelli (1967) estimates the temperatures of the dark mottles to be $T<10,000 \mathrm{~K}$. Based on some parameters given by the cloud model, Tsiropoula et al. (1993) derived values in the range 7100-13000 K. Later on, Tsiropoula \& Schmieder (1997) claimed that dark mottles for the microturbulent velocity around $10 \mathrm{~km} \mathrm{~s}^{-1}$ have $T=14,000 \mathrm{~K}$ with standard deviation $\approx 9200 \mathrm{~K}$ (see Table 1 of Tsiropoula $\&$ Schmieder 1997). The SMS temperature diagnostic suggests that the particular dark mottle analyzed here is at the lower end of previous temperature range estimates. Providing new insight, SMS suggests that the dark mottle is reasonably isothermal along its structure, at least up to $2 \mathrm{Mm}$ from its footpoint. However, more SMS dark mottle case studies will be required to actually understand how representative the present example is.

The application of SMS diagnostics to the mottle for lengths greater than $\sim 2 \mathrm{Mm}$ show a decrease in the plasma density and magnetic field gradients. Although these features were also found for an off-limb spicule (Verth et al. 2011), we point out that our estimates above $2 \mathrm{Mm}$ carry large uncertainties. The observed changes could be caused by the effects of the magnetic canopy. The merging flux tubes higher in the atmosphere could alter the rate at which the magnetic field decreases. At the canopy level, the flux tubes become more horizontal, which can change the density stratification along the structure. We note that the SMS estimates presented here are more applicable to the local plasma parameters of a particular small-scale flux tube, and they may not necessarily be considered as typical of all chromospheric structures. However, it has been demonstrated that by studying the variation of phase speed and transverse velocity of kink waves along mottles and fibrils, we can understand more completely the dominant plasma properties of chromospheric waveguides. Furthermore, a wealth of statistics for phase speed variations can provide typical values for the cut off period. Transverse oscillations that are ubiquitous in the chromosphere (Kuridze et al. 2012; Morton et al. 2012) are likely to be separated into propagating and non-propagating waves by the cut off period. Hence, it could be crucial to estimate how much kink wave energy is transported into the corona and what is trapped in the chromosphere.

This work is supported by the UK Science and Technology Facilities Council (STFC). R.E. acknowledges M. Kéray for patient encouragement and is also grateful to NSF Hungary (OTKA K83133). We thank the Air Force Office of Scientific Research, Air Force Material Command, USAF for sponsorship under grant No. FA8655-09-13085. G.V. acknowledges the support of the Leverhulme Trust.

\section{REFERENCES}

Andries, J., van Doorsselaere, T., Roberts, B., et al. 2009, SSRv, 149, 3 Aschwanden, M. J. 2004, in Physics of the Solar Corona (Berlin: Springer), 1283

Beckers, J. M. 1968, SoPh, 3, 367

Beckers, J. M. 1972, ARA\&A, 10, 73

Bogdan, T. J., Carlsson, M., Hansteen, V. H., et al. 2003, ApJ, 599, 626 Cavallini, F. 2006, SoPh, 236, 415

Centeno, R., Trujillo Bueno, J., \& Asensio Ramos, A. 2010, ApJ, 708, 1579 Crockett, P. J., Mathioudakis, M., Jess, D. B., et al. 2010, ApJL, 722, L188

De Pontieu, B., Carlsson, M., Rouppe van der Voort, L. H. M., et al. 2012, ApJL, 752, L12

De Pontieu, B., \& Erdélyi, R. 2006, RSPTA, 364, 383

De Pontieu, B., Erdélyi, R., \& James, S. P. 2004, Natur, 430, 536

De Pontieu, B., McIntosh, S. W., Carlsson, M., et al. 2007, Sci, 318, 1574 DeForest, C. E. 2007, ApJ, 661, 532

Eibe, M. T., Mein, P., Roudier, T., \& Faurobert, M. 2001, A\&A, 371, 1128

Edwin, P. M., \& Roberts, B. 1983, SoPh, 88, 179

Erdélyi, R. 2006, RSPTA, 364, 351 
Erdélyi, R., \& Fedun, V. 2007, Sci, 318, 1572

Finsterle, W., Jefferies, S. M., Cacciani, A., Rapex, P., \& McIntosh, S. W. 2004, ApJL, 613, L185

Fontenla, J. M., Balasubramaniam, K. S., \& Harder, J. 2007, ApJ, 667, 1243

Fujimura, D., \& Tsuneta, S. 2009, ApJ, 702, 1443

Giovanelli, R. G. 1967, AuJPh, 20, 81

Hansteen, V. H., De Pontieu, B., Rouppe van der Voort, L., van Noort, M., \& Carlsson, M. 2006, ApJL, 647, L73

Hasan, S. S., \& Kalkofen, W. 1999, ApJ, 519, 899

Hasan, S. S., Kalkofen, W., van Ballegooijen, A. A., \& Ulmschneider, P. 2003, ApJ, 585, 1138

He, J., Marsch, E., Tu, C., \& Tian, H. 2009a, ApJL, 705, L217

He, J., Tu, C., Marsch, E., et al. 2009b, A\&A, 497, 525

Hollweg, J. V. 1981, SoPh, 70, 25

Hollweg, J. V., Jackson, S., \& Galloway, D. 1982, SoPh, 75, 35

Jess, D. B., Mathioudakis, M., Christian, D. J., et al. 2010a, SoPh, 261, 363

Jess, D. B., Mathioudakis, M., Christian, D. J., Crockett, P. J., \& Keenan, F. P. 2010b, ApJL, 719, L134

Jess, D. B., Pascoe, D. J., Christian, D. J., et al. 2012, ApJL, 744, L5

Kneser, A. 1893, MatAn, 42, 409

Kukhianidze, V., Zaqarashvili, T. V., \& Khutsishvili, E. 2006, A\&A, 449, L35

Kuridze, D., Morton, R. J., Erdélyi, R., et al. 2012, ApJ, 750, 51

Kuridze, D., Zaqarashvili, T. V., Shergelashvili, B. M., \& Poedts, S. 2008, AnG, 26, 2983

Leenaarts, J., Carlsson, M., \& van der Voort, L. R. 2012, ApJ, 749, 136

Makita, M. 2003, PNAOJ, 7, 1

Mathioudakis, M., Jess, D. B., \& Erdélyi, R. 2013, SSRv, 175, 1

McIntosh, S. W., de Pontieu, B., Carlsson, M., et al. 2011, Natur, 475, 477

Morton, R. J. 2012, A\&A, 543, 6

Morton, R. J., Verth, G., Fedun, V., Shelyag, S., \& Erdélyi, R. 2013, ApJ, 768,17

Morton, R. J., Verth, G., Jess, D. B., et al. 2012, NatCo, 3, 1315

Okamoto, T. J., \& de Pontieu, B. 2011, ApJL, 736, L24

Pietarila, A., Aznar Cuadrado, R., Hirzberger, J., \& Solanki, S. K. 2011, ApJ, 739,92
Rimmele, T. R. 2004, Proc. SPIE, 5490, 34

Roberts, B. 1979, SoPh, 61, 23

Rosenthal, C. S., Bogdan, T. J., Carlsson, M., et al. 2002, ApJ, 564, 508

Rouppe van der Voort, L., De Pontieu, B., Hansteen, V., Carlsson, M., \& van Noort, M. 2007, ApJ, 660, 169

Rouppe van der Voort, L., Leenaarts, J., de Pontieu, B., Carlsson, M., \& Vissers, G. 2009, ApJ, 705, 272

Ruderman, M. S., \& Erdélyi, R. 2009, SSRv, 149, 199

Schunker, H., \& Cally, P. S. 2006, MNRAS, 372, 551

Scullion, E., Popescu, M. D., Banerjee, D., Doyle, J. G., \& Erdélyi, R. 2009, ApJ, 704, 1385

Sekse, D. H., Rouppe van der Voort, L., De Pontieu, B., \& Scullion, E. 2013, ApJ, 769,44

Solanki, S. K., \& Steiner, O. 1990, A\&A, 234, 519

Spruit, H. C. 1981, A\&A, 98, 155

Spruit, H. C. 1982, SoPh, 75, 3

Sterling, A. C. 2000, SoPh, 196, 79

Taroyan, Y., \& Erdélyi, R. 2009, SSRv, 149, 229

Trujillo Bueno, J., Merenda, L., Centeno, R., Collados, M., \& Landi DeglInnocenti, E. 2005, ApJL, 619, L191

Tsiropoula, G., Alissandrakis, C. E., \& Schmieder, B. 1993, A\&A 271,574

Tsiropoula, G., \& Schmieder, B. 1997, A\&A, 324, 1183

Tsiropoula, G., Tziotziou, K., Kontogiannis, I., et al. 2012, SSRv, 169, 181

Tziotziou, K., Tsiropoula, G., \& Mein, P. 2003, A\&A, 402, 361

Vernazza, J. E., Avrett, E. H., \& Loeser, R. 1981, ApJS, 45, 635

Verth, G., Goossens, M., \& He, J.-S. 2011, ApJL, 733, L15

Vissers, G., \& Rouppe van der Voort, L. 2012, ApJ, 750, 22

Wedemeyer-Böhm, S., Lagg, A., \& Nordlund, Å. 2009, SSRv, 144, 317

Wöger, F., von der Lühe, O., \& Reardon, K. 2008, A\&A, 488, 375

Zachariadis, Th. G., Dara, H. C., Alissandrakis, C. E., Koutchmy, S., \& Contikakis, C. 2001, SoPh, 202, 41

Zaqarashvili, T. V., \& Erdélyi, R. 2009, SSRv, 149, 355

Zaqarashvili, T. V., Khutsishvili, E., Kukhianidze, V., \& Ramishvili, G. 2007, A\&A, 474, 627 